\title{
Carnets
}

Revue électronique d'études françaises de l'APEF

Deuxième série - $10 \mid 2017$

Pratiques de l'errance, vécus de la mémoire

\section{La traduction comme déracinement et exil}

La version portugaise de La Vie devant soi, de Romain Gary

\section{Dominique Faria}

\section{OpenEdition}

Journals

Édition électronique

URL : http://journals.openedition.org/carnets/2259

DOI : $10.4000 /$ carnets.2259

ISSN : 1646-7698

Éditeur

APEF

Référence électronique

Dominique Faria, «La traduction comme déracinement et exil », Carnets [En ligne], Deuxième série -

10 | 2017, mis en ligne le 30 avril 2017, consulté le 19 avril 2019. URL : http://journals.openedition.org/ carnets/2259; DOI : 10.4000/carnets.2259

Ce document a été généré automatiquement le 19 avril 2019

\section{(c) (†) (8)}

Carnets est mis à disposition selon les termes de la licence Creative Commons - Atribution - Pas d'utilisation commerciale 4.0 International. 


\section{La traduction comme déracinement et exil}

La version portugaise de La Vie devant soi, de Romain Gary

Dominique Faria

\section{Texte traduit, texte exilé}

1 Rapprocher exil et traduction n'est pas nouveau. L'exil a déjà été employé comme un opérateur de lisibilité pour la traduction, une métaphore qui permet de mieux expliquer ce qui advient lorsqu'on traduit. Notamment par Walter Benjamin, qui conçoit la possibilité de l'existence d'une "pure langue » de la traduction, exilée dans les langues nationales ${ }^{1}$. Je me propose pour ma part d'approfondir cette analogie, partant de quelques aspects de l'exil qui ont également lieu lors de la publication d'un texte traduit.

2 Telle que je l'envisage ici, la traduction n'est donc pas l'activité mais le produit, elle n'est pas strictement linguistique, mais plutôt culturelle : il s'agit d'un texte qui quitte son pays d'origine pour s'installer ailleurs. Le texte traduit et l'exilé se disent dans une autre langue, intègrent une autre culture, à laquelle ils doivent s'adapter pour survivre. La traduction est le texte en exil. Elle est la survie du texte. Ce texte en survie doit désormais être interprété, redéfini par des regards étrangers porteurs de schémas d'interprétation du monde, de présupposés, qui diffèrent de ceux sous lesquels il a été créé.

Pourquoi rapprocher le texte traduit de l'exilé plutôt que de l'émigré ou l'expatrié ? Car, comme le signale Edward Saïd dans « Reflections on exile » (Saïd, 2000 : 181), si toutes ces modalités impliquent un départ du pays d'origine pour vivre ailleurs, l'exilé a la particularité d'être empêché de retourner et « une fois banni, l'exilé vit une vie anormale et misérable, stigmatisé en tant qu'étranger ${ }^{2}$. L'émigré et l'expatrié ont pris la décision de partir. Ils peuvent retourner au pays natal s'ils le souhaitent, un choix dont ne disposent nullement l'exilé et la traduction. Ce n'est que très exceptionnellement qu'un texte traduit retourne au pays d'origine, où il trouvera rarement des lecteurs. 
4 L'appartenance et l'identité s'avèrent être des enjeux majeurs tant dans l'exil que dans la traduction. Le déracinement déclenche un problème d'identité, car le texte traduit est autre. Les discours traditionnels le disent second et secondaire, lui refusent l'autonomie, ne lui reconnaissent pas une identité propre. Les idées, l'agencement des phrases, l'intrigue, les références culturelles sont ceux choisis par l'auteur de départ. Mais on les réécrit et on les inscrit dans le contexte d'arrivée. Est-ce le texte de l'auteur ou celui du traducteur? Quelle nationalité pour un texte traduit? Quel enracinement culturel? Ce que dit Talahite-Moodley à propos de l'exil s'applique aussi bien à la traduction: «l'expression de l'exil prend des formes multiples qui nuancent la notion d'enracinement dans une terre ou une culture singulière. Elle tend à contester une vision d'appartenance monolithique pour promouvoir des identités en devenir. » (Talahite-Moodley, $2007: 3$ ).

Comme conséquence - de ce déracinement, de ce manque d'appartenance, de cette « identité en devenir »- exilés et traduction subissent la méfiance de ceux qui se trouvent parfaitement installés du côté du texte original, des racines culturelles fixes, définissant traditionnellement l'individu et le texte. Il nous suffit de penser au statut du texte traduit par rapport au texte de départ et par rapport aux autres textes écrits directement dans la langue de traduction. Les préjugés abondent: on l'accuse de manque d'authenticité et d'originalité dans les deux contextes.

6 La notion de «belles infidèles » et la vieille formule "traduttore, traditore » exhibent directement cette méfiance. De même que les notions de fidélité et de liberté, que l'on retrouve déjà dans les premiers discours sur la traduction et qui sont à la base, de nos jours encore, de maintes réflexions sur la traduction, sous d'autres habits, comme les notions de domestication et de dépaysement. Une traduction retrouve toujours des détracteurs : si elle est perçue comme étant trop proche du contexte de départ, elle est infidèle à la langue et la culture d'accueil ; si elle est trop adaptée à son nouveau contexte on dit qu'elle trahit ses origines. Comme les exilés, les textes traduits sont envisagés comme des éternels étrangers. Comme étranges. Ils ne sont jamais effectivement chez eux nulle part.

7 Comme conséquence, ils éprouvent également des problèmes de reconnaissance et de statut. Antoine Berman signale que :

Les littératures étrangères traduites ne s'intègrent généralement pas à la littérature autochtone, sauf dans le cas de très grandes traductions (...) Elles restent des « littératures étrangères » même si elles marquent la littérature autochtone. (...) La littérature traduite ne s'intègre donc pas à la littérature autochtone, comme le montrent les rayons des librairies (Berman, $1995: 58$ ).

Les rayons des librairies dont parle Berman sont ceux où on isole généralement la littérature traduite. Ils symbolisent une problématique qui a des implications plus profondes. La vraie question pour moi est que le texte traduit n'est plus reconnu dans le pays d'origine comme intégrant le système littéraire national et il n'est reconnu non plus par le système d'accueil. Even-Zohar (2000) a beau avoir démontré l'existence d'un canon de la littérature traduite à l'intérieur de chaque système littéraire national, il n'en reste pas moins que ce canon, son influence sur la culture d'accueil, la qualité des ouvrages qui le composent demeurent, à quelques exceptions près, ignorés par les instances culturelles nationales.

9 Et ce, malgré les recherches et théories qui prônent, depuis les années 80 , l'inclusion de la traduction dans l'étude des littératures nationales. L'ouvrage traduit n'est évoqué ni dans les histoires des littératures dont il est issu, ni dans celles de la littérature d'accueil. Les 
traductions ne figurent nulle part. Elles sont ignorées par les discours qui fixent à la postérité la production littéraire et culturelle des peuples. Les histoires de la traduction nationales dans lesquelles on investit de plus en plus - du moins dans certains pays, comme la France ${ }^{3}$ et l'Espagne ${ }^{4}$ - rendent finalement compte des faits et de leurs effets, jusqu'ici plus ou moins méconnus. De même qu'il est crucial d'établir l'histoire d'exilés, immigrés et expatriés, pour peser le rôle des individus et des phénomènes qu'ils contribuent à créer, à l'intérieur de chaque histoire nationale, il est important de tenir compte des textes qui ont été traduits à chaque période, des traducteurs, des éditeurs et des maisons d'éditions qui s'en sont chargés, pour en dégager les conséquences sur les histoires des littératures nationales.

\section{La version portugaise de La vie devant soi : déracinement et appartenance}

10 Pour illustrer ces propos sur la traduction, on s'arrêtera sur la version portugaise de $L a$ vie devant soi, un roman publié par Romain Gary en 1975, sous le pseudonyme d'Émile Ajar. La problématique de l'identité et de l'appartenance, évoquées ci-dessus à propos de l'exil et de la traduction, se trouvent précisément au cœur de l'œuvre - et de la vie - de Romain Gary ${ }^{5}$. Outre sa fascination pour les pseudonymes, qui culmine dans sa double identité littéraire Romain Gary/Émile Ajar, ses origines mixtes y contribuent également : né en Lituanie, de père et mère juifs, il vit la plupart de sa vie en France.

Dans son œuvre, les personnages en quête d'identité ou à l'identité hybride abondent. Un des plus connus est Momo, le narrateur de La vie devant soí. Momo est un jeune Arabe, élevé par Madame Rosa, une ancienne prostituée juive, rescapée d'Auschwitz, qui garde des enfants de prostituées. Le scénario choisi est Belleville dans les années 70, un quartier caractérisé par une grande concentration d'émigrés et par sa multiethnicité. Momo n'a pas de mémoire de sa vie avant Belleville, ne connaît pas son père et garde peu de souvenirs de sa mère. Il est donc doublement déraciné : en termes culturels et familiaux. La seule réalité que ce personnage connaît est cette culture hétéroclite, cette communauté composée de gens aux origines et aux mœurs étrangers qui doivent vivre dans un nouveau contexte auquel ils ont du mal à s'adapter. Cette indéfinition identitaire du personnage est manifeste aussi par le fait qu'il oscille entre deux religions : il parle le yiddish et connaît les prières juives, mais il se dit un musulman, pratique le ramadan (VDS :53) et a appris le Coran.

Or, s'il est une spécificité à ce roman elle réside sans doute dans la voix de ce narrateur. On pourrait dire, à propos de ce texte de Gary, ce que dit Lise Gauvin $(2007: 6)$ à propos des écrivains francophones, chez qui on retrouve "une sensibilité plus grande à la problématique des langues, soit une surconscience linguistique qui fait de la langue un lieu de réflexion privilégié, un espace de fiction voire de friction. (...) Écrire devient alors un véritable "acte de langage". » Le français de Momo est celui qu'il a appris dans les rues de Belleville et celui des jeunes de son âge: on y trouve des incorrections, des idiosyncrasies et un langage oralisé et familier. Cette utilisation atypique du Français exhibe la marginalité du personnage-narrateur et dénonce simultanément les clichés d'un discours social, typique du monde des adultes qui l'entourent. La déviance de la norme linguistique correspond à une déviance par rapport à la norme sociale et, par conséquent, elle isole. C'est cet isolement, cette solitude qui hantent ce jeune Momo et 
qui expliquent sa peur du futur que le titre du roman met en exergue, et qui conditionnent la lecture du texte, à savoir sa peur face à l'idée d'avoir «la vie devant soi ». Le rapport que ce personnage entretient avec la langue française est donc indispensable pour le caractériser, d'autant plus que personne d'autre ne le décrit au long du roman. Elle exprime sa façon d'interpréter et de dire le monde.

Je m'intéresserai à la traduction portugaise de ce texte, Uma vida à sua frente $e^{7}$, faite par Joana Cabral et publiée en 2011, afin de cerner ce qui advient, lors de la traduction, de cette « surconscience linguistique » dont parle Lise Gauvin, et de réfléchir aux différences dans la caractérisation $d u$ personnage dans la version portugaise du texte, par comparaison avec la française. Je m'arrêterai sur trois traits particuliers du discours de ce personnage: le recours à un langage familier, les incorrections linguistiques et les idiosyncrasies.

Commençons par le langage familier. Lors de l'analyse de la traduction, on constate que la plupart de ce lexique est remplacé par des mots qui ont à peu près le même sens et qui appartiennent au même niveau de langue en portugais. Un grand nombre de ces vocables est toutefois remplacé par des mots qui appartiennent à un niveau de langue plus soutenu. C'est le cas des exemples suivants :

\begin{tabular}{|c|c|}
\hline se marrait (VDS: 18 ) & se ria $(V S F: 13)[\text { riait }]^{8}$ \\
\hline je les ai foutus... (VDS : 26) & mandei-os (VSF : 18) [je les ai envoyés] \\
\hline gueuler (VDS : 26) & ralhar (VSF : 18) [gronder] \\
\hline je les ai foutus... (VDS : 26$)$ & mandei-os (VSF : 18) [je les ai envoyés] \\
\hline costaud (VDS : 34$)$ & robusto (VSF : 23) [robuste] \\
\hline turlupinait (VDS : 35) & de todas as maneiras (VSF : 24) [de toutes les façons] \\
\hline faux jeton (VDS : 50) & Hipócritas (VSF : 34) [Hypocrites] \\
\hline chialer (VDS : 84) & chorar (VSF: 55) [pleurer] \\
\hline J'ai eu du pot (VDS : 203) & tive sorte (VSF : 136) [j'ai eu de la chance] \\
\hline qui en ont ralbol (VDS : 215) & que estão fartas (VSF : 145) [qui en ont marre] \\
\hline sa piaule (VDS : 27) & quarto (VSF : 18) [chambre] \\
\hline
\end{tabular}

La conséquence de ces choix, surtout parce qu'ils sont systématiques, est que le texte que le lecteur portugais lit est plus «normal», léger, fluide que celui que le lecteur de la version française connaît. Souvent, dans la version traduite, Momo utilise un vocabulaire qu'un jeune de son âge ne maîtrise généralement pas. C'est le cas de la traduction de « faux jeton " par " hipócrita " ( hypocrite » en français), ou de costaud par « robusto " (qui équivaudrait à « robuste » en français).

De même, la version portugaise est plus homogène, estompant le contraste entre le langage familier et la langue standard. Prenons comme exemple un paragraphe de la 
version française (VDS : 27-28), dans lequel trois mots différents sont utilisés pour se référer aux enfants : môme (qui surgit 3 fois), enfant(s) (3 fois) et mioche (utilisé une seule fois). Ils sont remplacés, dans la version portugaise par « filho(s) », qui est le mot en portugais standard pour « fils » et pour « enfant(s) », et une seule fois, par « miúda » (VSF : 18), une désignation plus familière pour « fille ». Or, il y a une différence subtile dans le choix des mots en français. Lorsque Momo parle de sa perception du monde, il utilise un langage plus familier : « Moi j'ai vu chez nous des mères pleurer, on les avait dénoncées à la police comme quoi elles avaient un môme dans le métier qu'elles faisaient et elles mouraient de peur » (VDS : 28). Quand il rapporte le discours ou la perception du monde des adultes, même si cela n'est pas explicitement indiqué dans le texte, il utilise plutôt «enfant»: «Elles [les prostituées] ont besoin de leurs enfants pour avoir raison de vivre. »(VDS : 28).

Un autre trait typique du discours de ce personnage est les incorrections linguistiques, notamment des constructions syntaxiques qui rendent le discours ambigu, un trait typique du langage enfantin que la traduction a tendance à corriger, comme on peut le vérifier par les exemples suivants :

\begin{tabular}{|l|l|}
\hline $\begin{array}{l}\text { ils étaient la plupart du temps } \\
\text { musulmans comme moi (VDS:34) }\end{array}$ & $\begin{array}{l}\text { A maioria era muçulmana como eu (VSF : 23) [La plupart } \\
\text { était des musulmans comme moi] }\end{array}$ \\
\hline $\begin{array}{l}\text { il avait les yeux comme si c'était pour } \\
\text { faire peur (VDS : 34) }\end{array}$ & $\begin{array}{l}\text { os olhos dele metiam medo (VSF :23) [Ses yeux faisaient } \\
\text { peur] }\end{array}$ \\
\hline $\begin{array}{l}\text { il se faisait les ongles chez les } \\
\text { manicures qui étaient roses aussi ( } \\
\text { VDS : 46) }\end{array}$ & $\begin{array}{l}\text { arranjava as unhas, rosas também, nas manicures (VSF } \\
: 31) \text { [il se faisait les ongles, roses elles aussi, chez les } \\
\text { manicures] }\end{array}$ \\
\hline $\begin{array}{l}\text { des danseuses blanches sur le dos de } \\
\text { chevaux en tutu (VDS:94) }\end{array}$ & $\begin{array}{l}\text { dançarinas brancas de tutu montadas a cavalo (VSF :63) } \\
\text { [des danseuses blanches en tutu sur le dos de chevaux] }\end{array}$ \\
\hline
\end{tabular}

18 Pour ce qui est des idiosyncrasies de ce personnage, une des plus singulières et récurrentes au long du texte est l'expression «comme j'ai l'honneur». Pierre Bayard (1990: 111) signale "une confusion entre les niveaux de langue de l'enfant et de l'adulte", qu'il dit être typique de ce roman. Ceci en est un bon exemple. L'enfant s'approprie une expression qui appartient au langage des adultes, lui donnant un autre sens. Or, dans la version portugaise, non seulement le portugais est très correct et très courant, mais l'expression n'est pas toujours traduite de la même façon, ce qui efface cette particularité. Les deux exemples suivants montrent précisément ceci : « comme j’ai eu l'honneur » (VDS : 52) est traduit par « como já vos contei » (VSF : 35) [comme je vous l'ai déjà raconté], mais « C'est comme j'ai l'honneur » (VDS : 82) est traduit par « Palavra » (VSF : 54), [croyez-moi]. La traductrice a interprété le sens attribué par l'enfant à ces mots dans chaque situation et l'a rendu par d'autres mots.

19 Avant de conclure, je m'arrêterai sur la traduction d'un mot qui a une importante charge culturelle, pour exemplifier comment un texte qui n'a pas été traduit selon une stratégie cibliste, doit quand même s'inscrire dans la culture d'accueil, comment un texte traduit est toujours condamné à devenir un texte en exil. Il s'agit de l'insulte "Sale bicot » (DVS : 15), utilisé en France pour insulter quelqu'un aux origines arabes et que la traductrice a 
traduit par «mouro » (VSF : 10). Ce mot, qui a la même racine que «maure » en français, désigne quelqu'un originaire du Maghreb et a la particularité de n'être plus utilisé dans le langage courant au Portugal depuis longtemps, contrairement à « sale bicot» en France. Le mot « mouro » est plutôt associé à un moment précis de l'histoire du Portugal, lors de la fondation de la nation. Les connotations de ces deux expressions diffèrent donc énormément. En traduction il n'y a jamais de solution parfaite, et souvent les mots ont des charges symboliques spécifiques dans chaque langue, car ils sont liés à des phénomènes sociaux et historiques différents.

\section{Conclusion}

La traduction est donc un texte en exil, un texte en survie, l'eternel étranger ayant perdu sa position au pays natal et auquel on accorde une place marginale dans le pays d'accueil. Son rôle crucial n'est pris en compte dans aucun des pays entre lesquels elle se partage. Alors que La vie devant soi appartient à la littérature française et on retrouve facilement dans les volumes concernant cette littérature des références à ce roman, un des plus célèbres de Romain Gary, Uma vida à sua frente, sa traduction portugaise, ne figurera probablement nulle part : les histoires de la littérature française l'ignorent et celles de la littérature portugaise aussi.

Une vie devant soi et Uma vida à sua frente ne sont pas exactement le même texte, écrit par le même auteur. Ils ne sont pas non plus des textes entièrement différents. Cette identité double, cette hybridité du texte traduit nourrit une méfiance séculaire. La traduction littéraire joue pourtant un rôle majeur dans la circulation des savoirs, dans les échanges culturels et dans la connaissance de l'Autre. Sans la version portugaise de ce roman de Gary, le lecteur portugais aurait moins de chances de connaitre le travail de cet auteur et la réalité sociale dont il trace le portrait. Et ce, malgré les disparités entre les deux versions du texte. Même si la version portugaise est plus correcte, même si elle estompe le contraste entre la langue standard des adultes et le langage familier et l'utilisation naïve qu'en fait un jeune aux origines arabes, élevé par une Juive, dans le Belleville des années 70 .

Si le regard qu'on porte sur un texte traduit comme sur l'exilé est toujours suspicieux, cela se doit à ce qu'ils ne sont pas monolithiques, stables et donc familiers et confortables. Ils déclenchent une problématique identitaire qui dérange tous ceux qui s'attachent à une vision traditionnelle du monde, tous ceux que l'altérité trouble.

\section{BIBLIOGRAPHIE}

AUDI, Paul (2007). Je me suis toujours été un autre : le paradis de Romain Gary. Paris : C. Bourgois éditeur. 
AUDI, Paul, Martin, Jean-Pierre (2007). « Gary, entre appartenance et identité », in Julien Roumette (dir.). Revue Littératures, Romain Gary, l'ombre de l'histoire, no 56, Presses Universitaires du Mirail, pp. 109-127.

BAYARD, Pierre (1990). Il était deux fois romain Gary. Paris : PUF.

BERMAN, Antoine (1995). Pour une critique des traductions : John Donne. Paris : Gallimard.

CRISTÓBAL, Enrique Pérez (2011). « L'exil comme métaphore de la traduction : la pure langue de Walter Benjamin et la langue vive de Carles Riba », Meta : journal des traducteurs / Meta : Translators' Journal, vol. 56, n 4, 2011, pp. 852-860.

EVEN-ZOLAR, Itamar (2000). «The Position of Translated Literature within the Literary Polysystem ", in Lawrence Venuti (ed.). The Translation Studies Reader. London \& New York : Routledge, 2000, pp. 192-197.

GARY, Romain (1975). La Vie devant soi. Paris : Mercure de France.

GARY, Romain (2011). Uma vida à sua frente. Porto : Sextante Editora.

GAUVIN, Lise (2007). Écrire pour qui ? L'écrivain francophone et ses publics. Paris : Karthala.

SAÏD, Edward (2000). Reflections on exile and other essays. Cambridge, Massachusetts : Harvard University Press.

\section{NOTES}

1. Voir Enrique Cristóbal (2011).

2. Je traduis.

3. Voir les quatre volumes de l'Histoire des traductions en langue française, publiés par les Éditions Verdier et coordonnés par Yves Chevrel et Jean-Yves Masson.

4. Voir l'Historia de la traducción en España, projet coordonné par Francisco Lafarga et Luis Pegenaute (URL: http://www.cervantesvirtual.com/obra/historia-de-la-traduccion-enespana--0/).

5. Comme le montrent d'ailleurs Paul Audi et Jean-Pierre Martin (2007). A ce propos, voir aussi Audi (2007).

6. Désigné dorénavant par VDS.

7. Que nous désignerons dorénavant par VSF.

8. Les exemples pris de la version portugaise seront suivis de leur retraduction en français, entre crochets.

\section{RÉSUMÉS}

L'exil fonctionne comme une métaphore qui permet de mieux saisir les enjeux majeurs qui soutiennent et la traduction et la réflexion théorique et critique qui la prend pour objet. Une approche de la traduction portugaise du roman de Romain Gary, entendue comme texte en exil sert dans cet article à illustrer nos propos. 
Exile serves as a metaphor to better grasp the major aspects underlying translation as well as the discourses and theories on translation. The Portuguese translation of Romain Gary' novel, taken as a text in exile, illustrates our previous remarks.

INDEX

Mots-clés : traduction, exil, littérature française, Portugal, roman français contemporain

Keywords : translation, exile, French literature, Portugal, French contemporary novel

\section{AUTEUR}

\section{DOMINIQUE FARIA}

Un. des Açores/CEC-Lisboa

dominique.ar.faria[at]uac.pt 